\title{
What is the global prevalence of dental healthcare needs and unmet dental needs among adolescents?
}

\author{
Paisi, Martha
}

http://hdl.handle.net/10026.1/18296

10.1038/s41432-020-0146-y

Evidence-Based Dentistry

Springer Science and Business Media LLC

All content in PEARL is protected by copyright law. Author manuscripts are made available in accordance with publisher policies. Please cite only the published version using the details provided on the item record or document. In the absence of an open licence (e.g. Creative Commons), permissions for further reuse of content should be sought from the publisher or author. 
S00-000

Summary Review / DENTAL PUBLIC HEALTH

\section{Title/Question}

What is the global prevalence of dental healthcare needs and unmet dental needs among adolescents?

\section{Authors}

Martha Paisi, ${ }^{1,2}$ Robert Witton, ${ }^{1,2}$ Philip Radford, ${ }^{3}$ Anastasios Plessas ${ }^{1}$

${ }^{1}$ Peninsula Dental School, University of Plymouth, Plymouth, United Kingdom.

martha.paisi@plymouth.ac.uk

robert.witton@plymouth.ac.uk

anastasios.plessas@plymouth.ac.uk

${ }^{2}$ Peninsula Dental Social Enterprise, University of Plymouth, Plymouth, United Kingdom.

robert.witton@plymouth.ac.uk

${ }^{3}$ Rotherham NHS Foundation Trust Community Dental Service, Barnsley, United Kingdom.

philip.radford2@nhs.net 


\section{A Commentary on}

Ghafari M, Bahadivand-Chegini S, Nadi T, Doosti-Irani A. The global prevalence of dental healthcare needs and unmet dental needs among adolescents: a systematic review and meta-analysis. Epidemiol Health. 2019;41:e2019046. doi: 10.4178/epih.e2019046.

\section{Data sources}

Databases searched included Web of Science, PubMed, and Scopus.

\section{Study selection}

Cross-sectional studies that estimated dental healthcare needs and unmet dental needs in young people, aged 10-19 years were considered for inclusion. No limitations in terms of year of publication, language, location of the study, gender and race of the participants, or the type of dental health needs and unmet needs were applied. The papers were screened on title and abstract and then on full text by two reviewers. Any disagreements were resolved through discussion and consultation with a third reviewer.

\section{Data extraction and synthesis}

Data were extracted by three reviewers. Critical appraisal was conducted by two reviewers using the Joanna Briggs Institute critical appraisal checklist. A PRISMA flow chart was used to present the study selection results. Summary measures on the prevalence of dental health needs and unmet needs were calculated. For the metaanalysis, the inverse variance method was used to obtain pooled summary measures. 


\section{Results}

Fifty seven studies were included in the review. The overall prevalence of dental healthcare needs was $49 \%$ (95\% Cl: $42-56)$ across all types of dental care. The highest prevalence was that for periodontal treatment needs (71\%; $95 \% \mathrm{Cl}: 46-96$ as reported in four studies), followed by that of general treatment needs $(59.0 \%$; $95 \% \mathrm{Cl}: 42-75$ as report in 12 studies), orthodontic treatment (46\%; $95 \% \mathrm{Cl}: 38-53$ as reported in 32 studies), and lastly that of malocclusion treatment needs (39\%; $95 \% \mathrm{Cl}: 28-50$ as reported in nine studies). The pooled prevalence of unmet dental needs as reported in nine studies was 34\% (95\% Cl: 27-40) with the highest prevalence found in Southeast Asia (72.3\%; 95\% Cl: 70.1-74.5) and the lowest one in Europe (11.8\%; 95\% Cl: 3.420.3).

\section{Conclusions}

The results of this review showed that the prevalence of dental healthcare needs was higher in America and Europe while unmet needs were more prevalent in Southeast Asia and Africa. The former could partly be explained by the lower number and sample sizes of studies conducted in developing countries.

GRADE Rating: Low (one out of four EBD stars) 


\section{Commentary}

Dental diseases remain the most common noncommunicable diseases (NCDs) ${ }^{1}$. They can negatively affect quality of life, and are the fourth most expensive to treat. ${ }^{2}$ While significant improvements in population's oral health have taken place over the past four decades, inequalities both between and within countries have persisted and/or widened. ${ }^{3,4}$ The Social Determinants of Health Framework ${ }^{5}$ highlights that the conditions that people 'are born, live, work and age and the unequal distribution of power, money and resources' influence people's decisions and choices, and are what drive inequalities. Although, the disproportionate differences in oral health across different groups are due to an interaction of a number of factors many of which are beyond an individual's control, dental service utilisation is an important indicator of oral health status and has a role to play in these disparities. ${ }^{6}$

The aim of this systematic review and meta-analysis was to examine the prevalence of dental healthcare needs and unmet dental needs among adolescents. The authors defined unmet needs as 'the difference between the healthcare services required to cope with a health problem and the services received'. After duplicates were removed and articles screened on title/abstract and full text level, 57 studies were included in the review. The age of the participants ranged from six to twenty years and thus deviated from the original inclusion criteria of 10-19 years. When considering all types of dental care, approximately $50 \%$ of the participants worldwide were found to require dental treatment, while $34 \%$ had unmet dental needs. 
The protocol was not registered a priory on any database. The PRISMA guidelines were followed for the conduct and reporting of the review. The authors searched only online databases of information, and as a result there is an increased risk of publication bias. Furthermore, no experts were consulted and relevant studies may have been missed. The eligibility criteria for the inclusion of studies were not clearly defined. It is unclear whether the data extraction form was pilot-tested. Taking into account the potential impact of quality assessment on the synthesis and interpretation of results, information on how critical appraisal was used to inform the findings, would have strengthened confidence in the review's conclusions. The findings of the review may have been affected by the over representation of surveys from the orthodontic literature. In addition, the results of periodontal treatment needs was based on only four studies (less than 4500 participants). As acknowledged by the authors, the systematic review and meta-analysis was limited by high heterogeneity due to the low quality of some of the included studies, and the use of different tools and criteria to detect dental healthcare needs.

The review showed that the highest prevalence of unmet dental needs among adolescents was in Southeast Asia while the lowest prevalence was in Europe. This could be suggestive of low utilisation of healthcare services in the Southeast Asia region, due perhaps to low capacity of healthcare systems, or cost of dental treatment. Studies exploring the reasons for these disparities in service utilisation are warranted. Furthermore, efforts are needed to identify means to improve access to, and utilisation of dental services in this region. Considering that oral diseases and other non- 
S00-000

communicable conditions share common modifiable risk factors, an integrated approach to address these common risk factors is imperative in tackling the burden of both oral diseases and other NCD. ${ }^{2}$

\section{Practice Points}

As part of an action plan to reduce inequalities, policy makers and dental professionals should improve access to dental care for disadvantaged populations and strengthen their wider social environment.

Public health interventions targeting common risk factors through a life-course approach have a great potential to reduce the impact of oral disease and unmet dental treatment needs.

\section{References}

1)WHO. Oral Health. Geneva: World Health Organisation; 2018. Available from: https://www.who.int/news-room/fact-sheets/detail/oral-health (Accessed February 2020)

2)NCD Alliance and FDI World Dental Federation. Accelerating action on oral health and NCDs. Achieving an integrated response. Geneva: FDI World Dental Federation, nd.

3) Murray JJ, Vernazza CR, Holmes RD. Forty years of national surveys: an overview of children's dental health from 1973-2013. British Dental Journal 2015;219:281-285.

4) Do LG. Distribution of caries in children: variations between and within populations. Journal of Dental Research 2012;91:536-543.

5) Commission on Social Determinants of Health. Closing the gap in a generation: health equity through action on the social determinants of health. Final report of the Commission on Social Determinants of Health. Geneva: World Health Organisation, 2008.

6) Raison $\mathrm{H}$, Harris RV. Interventions to reduce socio-economic inequalities in dental service utilisation - a systematic review. Community Dental Health 2019; 36(1):39-45. 